\title{
ORIGEN Y DESARROLLO DE LOS HÁBITOS Y COSTUM- BRES ALIMENTARIAS COMO RECURSO SOCIOCULTURAL DEL SER HUMANO: Una aproximación a la Historia y Antropología de los cuidados en la Alimentación
}

\author{
Manuel Lillo Crespo*, Mª Flores Vizcaya Moreno**
}

*D.U.E. Licenciado en Enfermería. Licenciado en Antropología Social y Cultural. Miembro de la Asociación de Historia y Antropología de los cuidados enfermeros de la Universidad de Alicante.

**D.U.E. Licenciada en Antropología Social y Cultural. Ayudante de Escuela universitaria del Departamento de Enfermería, Universidad de Alicante. Miembro de la Asociación de Historia y Antropología de los cuidados enfermeros de la Universidad de Alicante.

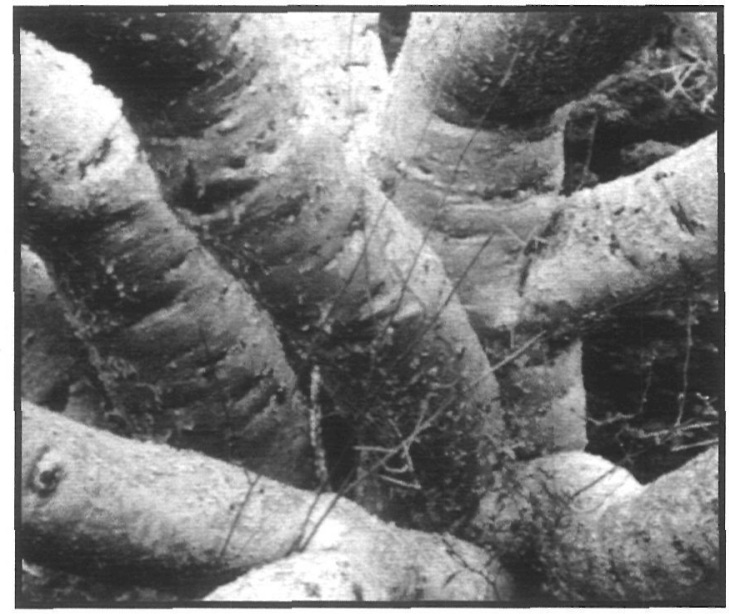

ORIGEN AND DEVELOPMENT OF ALIMENTARY HABITS AND CUSTOMS AS A SOCIOCULTURAL RESOURCE FOR MANKIND: An approach to History and Anthropology of care with regard to alimentation.

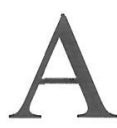
mongst all creatures on earth, man is the only one that has a "sense of destiny" and is able to symbolise even what appears to be strictly physical and material objects or phenomena. Therefore, any food -fruits, vegetables, pulses, fish- can possess various symbolic values according to different cultures and eras. The characteristics that link mankind with animals, and the social organisations and abilities developed long ago constitute a precious legacy which -in slow, quiet but constant evolution- have survived until present. The role that food has played within human diet, the involuntary nutrition process and the conscious feeding action is approached here from an anthropological point of view, following a clear historic view.

The main objective of this work is to show the way in which the social and symbolic value of food through history in different cultures has survived, where its starting point is located, and the foundations on which its essence with regard to care leans.

\section{INTRODUCCIÓN}

ntre todas las criaturas de la Tierra, el hombre es la única que posee el sentido del desL tino y el que puede simbolizar incluso sobre aquello que a simple vista pudiese parecer un fenómeno u objeto estrictamente físico y material. Así, cualquier tipo de alimento -frutas, hortalizas, legumbres, pescado...- goza de distintos valores simbólicos atribuidos por el hombre de distintas culturas en diferentes épocas, ya que los caracteres que unen al hombre con los animales, así como las habilidades y las organizaciones sociales que desarrolló mucho antes de que empezaran las épocas históricas, constituyen un preciado legado que aunque en lenta, silenciosa, pero sin embargo constante evolución, se ha perpetuado hasta los tiempos actuales. El papel que los alimentos han jugado dentro de la dieta humana, así como en el proceso involuntario de nutrición y en la acción consciente de alimentarse, se traduce en este trabajo desde su vertiente antropológica, siguiendo una clara visión histórica. 
El objetivo principal de este trabajo es mostrar de qué forma ha perdurado ese valor simbólico y social de los alimentos a lo largo de la Historia en diferentes culturas, dónde se encuentra su punto de partida y cuáles son los pilares sobre los que descansa su esencia en relación a los cuidados.

\section{MATERIAL Y MÉTODOS}

En primer lugar se ha llevado a cabo una interpretación del objeto antropológico de estudio: los cuidados en la alimentación desde los albores de la creación, empleando para ello los usos interpretativos propios de la antropología física y evolutiva, teniendo en cuenta la evolución del hombre en todos sus aspectos desde la prehistoria y en concreto con el descubrimiento del fuego - como medio impulsor de la tradición culinaria -. También se ha planteado la reinterpretación de la figura de la mujer como cuidadora esencial en el terreno doméstico y en concreto dentro del campo de la alimentación y la dieta familiar en diferentes culturas.

\section{DESARROLLO}

Desde una perspectiva social podemos afirmar que los alimentos representan mucho más que un recurso para el proceso de nutrición. De hecho, en todas las sociedades humanas, los alimentos juegan muchos y diferentes papeles, ya que se encuentran indiscutiblemente encastrados dentro de los aspectos religiosos, económicos y sociales de la vida diaria. Para los integrantes de las distintas culturas, los alimentos son interpretados como una amplia gama de símbolos, expresando y creando relaciones entre hombres, entre hombre y mujer, entre el propio hombre y los dioses, con la naturaleza, etc... Por lo tanto, podemos afirmar que los alimentos constituyen una parte esencial de la forma en que determinadas comunidades humanas se organizan y funcionan, así como de la forma en la que sus integrantes miran al mundo y su diversidad.

E1 famoso antropólogo Claude Lévi-Strauss (1964) aclaraba en su obra "Lo crudo y lo cocido" que así como no existe ningún grupo humano que no tenga una lengua hablada, tampoco existe ninguna comunidad actualmente que de alguna forma no cocine los alimentos, en mayor o menor medida. De hecho, la constante transformación de los alimentos crudos en alimentos cocinados no hace más que expresar el posible paralelismo entre 10 natural y lo cultural.

Resulta curioso observar las marcadas diferencias entre las creencias y las prácticas de distintas sociedades humanas en torno a los alimentos, ya que existe una amplia variedad de sustancias que son consideradas alimentos por unos grupos y no lo son para otros. Alimentos que suponen una fuente de nutrientes para algunas comunidades, están completamente prohibidos en otras. De hecho, hay claras diferencias entre las formas de preparar, cocinar y almacenar un mismo tipo de alimento en distintas culturas.

El descubrimiento del útil arte de cocinar posiblemente esté estrechamente relacionado con la afición del hombre primitivo por la sabrosa médula que contienen los huesos de gran tamaño. Cuando se aplasta un hueso crudo con una piedra, resulta difícil separar la médula; pero cuando el hueso se calienta, la médula se extrae con toda facilidad. Si se hierve, la médula se ablanda y se puede chupar o bien tirar de ella con los dientes. En los restos dejados por el hombre de Neandertal, los largos huesos de los animales aparecen rajados o astillados, pero no existen pruebas de que se hirvieran para ablandar la médula.

Según los investigadores, en algún momento de su vida, el hombre comenzó a guisar. Pronto debió descubrir que cocinando podía conseguir algo más que desprender la médula de los huesos: ablandar las duras fibras de la carne y de las raíces, facilitar su masticación y reducir considerablemente el tiempo destinado a la comida. Quizá estos descubrimientos básicos fueron los que permitieron al hombre prehistórico emprender una experiencia verdaderamente humana. Algunos estudios realizados en los parientes del hombre, por ejemplo los gibones, ponen de manifiesto que dedican la mitad de sus horas de vigilia a comer, y la otra mitad a ir y venir desde los lugares donde comen hasta las ramas de los árboles donde reposan, lo cual resulta sorprendente si tenemos en cuenta que toda su actividad social se ve condicionada por el fenómeno de la alimentación. Los gorilas, que se alimentan de tallos de bambú y de otros vegetales, emplean 
en la tarea de comer y masticar la mayor parte de las horas de luz diurna. El hombre primitivo, durante los primeros tiempos de la Edad de Piedra, por robustas que fueran sus mandíbulas o por muy largos que tuviera los dientes, hubo de pasar por los mismos inconvenientes. Pero tan pronto como empezó a cocer sus alimentos pudo realizar la tarea de comer en unas pocas horas y disponer del resto del día para cazar, construir herramientas y dedicarse a otros quehaceres. El hecho de cocinar los alimentos también estuvo relacionado con la distribución de las tareas dentro de la familia, quedando ésta relegada al mundo femenino junto a la recolecta de las cosechas, el cuidado de los niños y la atención a los ancianos, así como otras tareas domésticas. Es la mujer quien, una vez más, asume el papel de cocinar y la necesidad de alimentarse, dentro del universo de los cuidados. Es a partir de esta etapa cuando podemos decir que las necesidades primordiales de comer y beber no son iguales en el animal y en el hombre, es decir, no resultan un denominador común al hombre y al animal. El hombre con esta nueva conducta se distingue de los demás animales: por eso se convierte en un ser gastrónomo por excelencia.

"Nuestra necesidad de alimentarnos no se da en una forma puramente instintiva. Comemos con la fantasía y con la razón, no menos que con la boca y el estómago. Ello abre las puertas a la economia y a la moral, pero antes que nada a la gastronomía. El hombre es un animal gastrónomo, en el sentido más radical de la palabra. Y justo porque lo es, tienen sentido las virtudes morales de la abstinencia y de la sobriedad, por una parte, y por la otra, todo ese sector de la vida económica que constituye el ramo de la alimentación. Nada de ello, en efecto, tendría lugar si esta necesidad nos fuese dada - como ocurre en los otros animales de una manera instintiva. La economía y la moral de la alimentación presuponen, por tanto, la gastronomía como la forma en la que, ya en su necesidad de alimentarse, es el hombre algo más que un animal".

Antonio Millan Puelles

De hecho, no existió una preocupación excesiva por regular la alimentación diaria en las culturas precolombinas, salvo quizá en las clases altas. Se comía deprisa, sin ayuda de utensilios y con escasa preparación de los platos. Afortunadamente, el tomate y el chile hacían milagros para mejorar los sabores. Para los esquimales no había una hora de comer. En realidad, no tenían hora para nada, porque las noches duraban cuatro meses y los días otros cuatro. Se comía cuando se tenía hambre o cuando se cazaba, y con auténtica gula. El esquimal podía y puede comerse cuatro o cinco kilos de carne de una sola vez sin sentir la menor molestia estomacal. En la línea de lo comentado hasta el momento es importante destacar que existen alimentos considerados comestibles por unas comunidades humanas y no comestibles por otras; alimentos considerados religiosos por unos y profanos por otros; alimentos que actúan con poder terapéutico para algunos y que otros ni siquiera se atreverían a ingerirlos... pero ¿quién determina todas estas diferencias?. Mientras que en el Hinduismo está prohibido matar y comer cualquier tipo de animal, particularmente la vaca; la leche y sus derivados pueden consumirse -ya que no se considera que intervengan en el proceso vital del animal-; el pescado y los huevos son comidos con poca frecuencia. Sin embargo para los seguidores del Judaísmo, los productos porcinos no pueden comerse y tampoco el pescado sin escamas y las aves de presa. Sólo los animales desangrados, limpios, que hayan superado el ritual de matanza -denominado Kosher- pueden comerse. Por otra parte la leche y la carne nunca deben mezclarse dentro de la misma comida. Lo cierto es que podríamos continuar dando ejemplos comparativos e ilustrativos acerca de las distancias entre culturas con respecto a la alimentación, sin embargo es preciso reflexionar sobre aquellas profesiones, como es el caso de la Enfermería, donde se da el choque cultural entre el valor simbólico que los alimentos tienen para el propio profesional y para el paciente o usuario de la institución sanitaria. Quizás la capacidad de afrontar situaciones culturalmente comprometidas como el mero hecho de cuidar la salud de personas con costumbres y hábitos alimentarios distintos es una de las razones que explican el que la Enfermería como disciplina se encuentre asentada en la Universidad, luche por nuevos objetivos y tenga una proyección cada vez más extensa del cuidar. A esta capacidad se la ha 
llamado "sensibilidad cultural" y de ella nos hablan las teorías actuales sobre la Competencia Cultural. Mientras que los cuidadores informales dentro de la familia -cuidados no remunerados, sin especialidad ninguna- se enfrentan a situaciones culturalmente asumidas: iguales costumbres alimentarias, de higiene, de comunicación, de sueño, etc; los profesionales de los cuidados no sólo cuidan de hábitos distintos a los propios sino también de la "cultura" del otro.

Es por todos estos hechos por los que podemos adivinar que la acción de cocinar y guisar determinó el papel social de los alimentos tal y como se ha entendido hasta el momento actual, ya que desaparece la función de sobrevivir unida a la de nutrirse. Es a partir de aquí cuando podemos comenzar a hablar de alimentos sociales, es decir, aquellos que se consumen en presencia de otras personas y que tienen un valor simbólico a la vez que nutricional dependiendo del contexto cultural en el cual se lleve a cabo. En todas y cada una de las sociedades humanas los alimentos se presentan como una forma de expresión, de creación y de relación entre individuos. Estas relaciones se establecen entre miembros de grupos con carácter religioso, étnico o social, y es por ello que los alimentos tendrán las propiedades de símbolos rituales. Así, cuando los alimentos se consumen en comunidad, llevan consigo muchas asociaciones que nos dirán la forma en la que unos participantes ven a los otros y al mundo que les rodea. Como todos los rituales, para los de alimentación existen unas normas pertenecientes a la cultura o grupo en particular. Estas normas o reglas determinarán quien prepara y sirve la comida, quienes pueden sentarse a comer juntos e incluso quien debe recoger el montaje más tarde. También se determina el tiempo y la preparación estética de los alimentos, el orden de los distintos platos a degustar, los utensilios a emplear, la forma en la que determinados productos deben consumirse o los "modales en la mesa". Los alimentos en sí están sujetos a patrones culturales que determinarán su tamaño, forma, consistencia, color, olor y sabor; lo cual los hará más idóneos para uno u otro acontecimiento sociocultural.

El alimento físico se carga de un valor simbólico radical cuando figura como una realidad material que nos remite a algo inasible pero real: a los aspectos profundos de la existencia humana. Al símbolo radical no se le puede pedir que explique científicamente lo que simboliza; pero naturalmente nos lleva más allá de sí mismo. Como símbolo radical, el alimento no es un signo convencional: aporta una realidad natural e intrínseca, es decir, refleja realmente lo significado. Una sociedad que no posee dichos símbolos radicales, podemos afirmar que se encuentra totalmente muerta desde una perspectiva cultural.

\section{CONCLUSIONES}

Desde una perspectiva sociocultural, se ha pretendido mostrar la gran diversidad de roles o papeles que los alimentos han jugado en las sociedades humanas, ya sea marcando el estatus socioeconómico, la ocupación, las subculturas de género, las culturas de identidad, creando relaciones sociales, expresando cambios vitales importantes, aniversarios, fiestas, reafirmando los valores religiosos, étnicos y regionales de un grupo... Debido a la gran variedad de roles, las prácticas y creencias dietéticas suelen ser difíciles de clasificar y estudiar, incluso cuando son peligrosas para la salud física.

En la actualidad existe un gran interés por los temas relativos a la nutrición, dietética y la alimentación en general. Este interés se circunscribe, sobre todo, a dos campos considerablemente diferenciados entre sí: por una parte el de la nutrición y dietética y por otra el de la tecnología de la producción de los alimentos, que a su vez, contienen también dos preocupaciones esenciales de cualquier país: la salud y la economía. Este interés desbordado por la visión economicista de la dietética y alimentación puede ignorar muchas de las vertientes que configuran un fenómeno tan complejo como es el de las pautas alimentarias de una sociedad, desde una perspectiva sanitaria. Ignorar esas concomitancias puede provocar la incomprensión, o una comprensión incorrecta, de determinadas conductas alimentarias, calificadas, desde una visión etnocéntrica de irracionales, dietética y/ o económicamente, por el solo hecho de no haber sabido captar su racionalidad. También esa misma ignorancia puede provocar el fracaso de determinadas actuaciones, en el campo del desarrollo de la Enfermería, la Salud Pública o en el económico de 
toda una comunidad, y conducir a la inhibición de los organismos públicos en relación a los contenidos de la publicidad, los hábitos y costumbres de los alimentos, hasta el punto de hacer inútil una política de prevención sanitaria cuyos objetivos estén en contradicción con determinados mensajes publicitarios. Conocer estos hábitos y costumbres de personas ajenas que en un momento dado comienzan a formar parte de nuestra entorno - ya sea en un hospital, un centro de salud o en nuestra propia vida privada - podría abrir muchas puertas a otros trabajos de tipo político, económico y social, con la finalidad de mejorar la competencia y el liderazgo de la profesión enfermera, centrándose en una competencia no sólo técnica sino también cultural. Llegados a este punto de la discusión, nos planteamos: ¿de qué forma se integran las personas que asisten a los servicios sanitarios dentro de las culturas de identidad que los profesionales sanitarios crean?, ¿qué función juega la alimentación como factor integrador dentro del sistema sanitario?, ¿qué hábitos o costumbres alimentarias se ven obligados a adoptar los usuarios del sistema cuando penetran en éste?, ¿qué es lo que conocemos de las costumbres propias de los usuarios de los servicios sanitarios?, ¿qué podemos aportarles?; estas son algunas de las cuestiones que subyacen de este discurso donde la Alimentación se convierte en el medio principal para llegar a su respuesta. Los estudios sociales centrados en la alimentación, así como su aplicación a la clínica pueden aportar nuevas pautas de actuación para la profesión enfermera, en la constante búsqueda de satisfacer las necesidades, en este caso de alimentarse, del ser humano. Sin embargo, no podemos ser rigurosamente científicos y al mismo tiempo hacer una interpretación de los datos observados en los usuarios de los servicios sanitarios sin antes hacer un estudio retrospectivo y echar la vista atrás para comprender los cauces por los que se ha llegado a lo que hoy contemplamos, haciendo a su vez una interpretación histórica y antropológica de los hechos. Es por ello, que con este trabajo se sugiere abrir un debate sobre este tema para que desde otros contextos y desde diferentes teorías y modelos de intervención como por ejemplo el de la competencia cultural del profesional de los cuidados se responda de manera efectiva a los interrogantes identificados.
"Y es que los hábitos y costumbres alimentarios no sólo suponen un recurso sociocultural para el ser humano como tal, sino también para el profesional de Enfermeria en su intento por demostrar su valia y liderazgo en el área de la salud".

Los autores.

\section{BIBLIOGRAFÍA}

HARRIS, M. (1985) Good to eat. Simon \& Schuster Ed, Nueva York.

LÉVI-STRAUSS, C. (1964) Les Mythologiques: I Le cru et le cuit. Ed. Plon, Paris.

LÉVI-STRAUSS, C. (1967) Les Mythologiques: II Du miel aux cendres. Ed. Plon, Paris.

LÉVI-STRAUSS, C. (1968) Les Mythologiques: III L'origine des manières de table. Ed. Plon, Paris.

ARSUAGA, J.L. (1999) El collar del Neandertal. Ed. Temas de Hoy, Madrid.

WATTERS, J.A. (1961) The epic of man. Time Incorporated, Nueva York.

ARDREY, R. (1983) La evolución del hombre. La hipótesis del cazador. Alianza $3^{\mathrm{a}}$ ed., Madrid.

CRUZ, J. (1991) Alimentación y Cultura. Antropología de la conducta alimentaria. Ed. EUNSA, Pamplona.

LUCENA, M. (1990) América 1492. Retrato de un continente hace quinientos años. Grupo Anaya S.A., Madrid.

FARB, P y ARMELAGOS, G (1980) Consuming Passions: The Anthropology of eating. Houghton Mifflin, Boston.

SPECTOR, R.E. (1985) Cultural diversity in health and illness. Appleton-Century-Crofts, Norwalk CT.

NEWBERNE, P.M. (1978) Diet and nutrition. Bull, New York Acad. Med. 54, 385-396, Nueva York.

CONTRERAS, J. (1993) Antropología de la Alimentación. Ed. Eudema, Madrid.

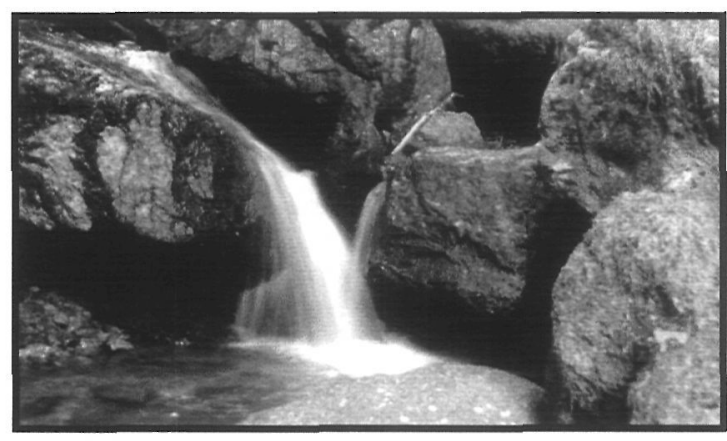

